Justyna Duch-Adamczyk

Adam-Mickiewicz-Universität, Poznań

DOI: $10.19195 / 0435-5865.142 .11$

\title{
Routineformeln im Presseinterview mit Prominenten am Beispiel des Magazins »Der Spiegel«
}

\section{Einführung}

Der vorliegende Aufsatz setzt sich zum Ziel, sprachliche Besonderheiten von Interviews mit Prominenten am Beispiel des Magazins »Der Spiegel« darzustellen. Dabei konzentriere ich mich auf die Präsentation bestimmter formelhafter Formulierungen, die typisch für Presseinterviews sind und bestimmten pragmatischen Funktionen wie etwa dem Gesprächsverlauf, dem Sprecherwechsel oder der thematischen Steuerung dienen. Im Fokus des Beitrags stehen also Formeln, die besonders für Medieninterviews prädestiniert sind. Dazu wird die pragmatische Funktion solcher Formeln ermittelt und näher bestimmt.

Zunächst gehe ich auf formelhafte Ausdrücke und Routinemittel in den Texten ein. Da Presseinterviews mündlich realisiert werden, aber schriftlich erscheinen, ist in diesem Kontext auch die Klärung der Termini Sprache der Nähe und Sprache der Distanz bzw. konzeptionelle Mündlichkeit / Schriftlichkeit und die Differenzierung geschriebene vs. gesprochene Sprache unerlässlich. Anschließend werden Merkmale des Interviews unter besonderer Berücksichtigung des Presseinterviews mit Prominenten präsentiert und dann ausgewählte, für Interviews charakteristische Formeln unter dem Aspekt ihrer Funktion im Text untersucht. Dazu werden 25 Interviews mit Prominenten herangezogen, die 2014 im Magazin »Der Spiegel« erschienen. In dem vorliegenden Beitrag beschränke ich mich auf ausgewählte sprachliche Mittel, die als für diese Textsorte charakteristisch und typisch gelten. Abschließend werte ich die Ergebnisse der Untersuchung aus und fasse sie zusammen. Anzumerken ist dazu, dass die durchgeführte Analyse sowie die Darstellung der Formeln für den untersuchten Bereich keinen Anspruch auf Vollständigkeit erheben. 


\section{Formelhaftes und Routine in Texten}

Sprechen ist an gewissen Normen und Standards orientiertes menschliches Handeln. Bei der Verfassung mündlicher oder schriftlicher Texte sind mehrere Normen einzuhalten, um die pragmatischen Funktionen etwa des Verlaufs und der Organisation des Gespräches zu wahren. Obwohl Sprechen als menschliches Handeln normativ reguliert ist, können Gebrauchsnormen oder -standards umstritten sein und verschieden erfasst und verstanden werden: So können im Verlauf eines Gespräches Erwartungen und Toleranzen variieren und sich in dessen Verlauf ändern. Für das Gespräch sind aus gebrauchsorientierter Sicht die Sicherung der Verständigung zwischen den Gesprächspartnern, die Progression des Gesprächsverlaufs sowie die sog. Image-Wahrung von primärer Bedeutung (vgl. Deppermann / Helmer 2013: 112 ff.). In dem die Grundlage des Interviews bildenden Gespräch gibt es Funktionen und Intentionen, die mit sprachlichen Mitteln realisiert oder zumindest verdeutlicht werden.

Der Gesprächsanalyse und sprachpragmatischen Untersuchungen wird seit der sog. kommunikativ-pragmatischen Wende zunehmende Aufmerksamkeit geschenkt. Mit der kommunikativ-pragmatisch ausgerichteten Sprachwissenschaft erfolgt ein Paradigmenwechsel von systemorientierter zu funktional orientierter Sprachwissenschaft (vgl. Heinemann / Vieweger 1991: 22) und Verlagerung des Schwerpunktes der Untersuchungen auf die Funktion ausgewählter Aspekte der Kommunikation. So werden auch geschriebene und gesprochene Texte pragmatisch und textlinguistisch untersucht. Interviews sind dabei ein besonders ergiebiger Forschungsgegenstand, weil in ihnen Elemente der gesprochenen und geschriebenen Sprache zusammenwirken. Zudem steht fest, dass das Verfassen von Interviews bestimmten Normen und Standards folgt, denn sie ,sind ritualisierte sprachliche Rollenspiele, bei denen die Rollen relativ strikt definiert und komplementär verteilt sind" (Sökeland 2003: 351). In dieser Hinsicht ist also anzunehmen, dass Interviews reich an ritualisierten und formelhaften Ausdrücken sind, durch die sich dialogische Texte auszeichnen und die von den Kommunikationspartnern als fest gewordene bzw. fertige Einheiten abgerufen werden können (vgl. Stein 1995: 130). Somit kann in Bezug auf Interviews die Rede von sprachlichen Routinen sein, die sich implizit auf das Handeln der Sprecher beziehen und die gegenseitigen Erwartungen der Kommunikationspartner steuern. Sie sind damit ein Teil des sozialen Handelns und erscheinen auf allen sprachlichen Ebenen. Die sprachlichen Routinen gewährleisten somit eine störungsfreie und dem gegebenen Kontext pragmatisch angemessene Sprachproduktion (Feilke 2012: 2 ff.).

Die Untersuchung der gesprächsspezifischen Formeln im Rahmen des vorliegenden Aufsatzes setzt die Formelhaftigkeit und Routiniertheit des Textes voraus. $\mathrm{Zu}$ diesen stellt Stein (2004: 262) fest:

Die Textproduktion unter Bedingung konzeptioneller Mündlichkeit basiert [...] auf einer Reihe von Routinen: Kompetente Sprecher können ohne größeren Verbrauch von Planungsressourcen auf mehr oder weniger feste Äußerungsteile und -strukturen zurückgreifen, um bestimmte kommunikative Aufgaben und Handlungen (wie Eröffnung und Beendigung von Gesprächen, 
Themenbearbeitung und Themenwechsel, Durchführung von Korrekturen/Reparaturen, Kommentierung von Äußerungen usw.) [...] zu bewältigen.

Bei der Verwendung gesprächsspezifischer Formeln sind also kommunikative Normen zu beachten, die für die gegebene Textsorte, in unserem Fall Interviews, typisch sind. Formulierungsroutinen sind zwar vor allem der gesprochenen Sprache eigen, finden aber auch in bestimmten Textsorten der schriftlichen Kommunikation häufige Verwendung (vgl. Feilke 2012: 6).

\section{Interview im Modell von Koch und Oesterreicher (1985, 2007)}

Die im Mittelpunkt dieses Beitrags stehenden Interviews des Magazins »Der Spiegel« werden mündlich realisiert und richten sich vor allem nach den Parametern gesprochener Sprache. Dabei verstehe ich nach Schwitalla (1997: 15) unter dem Terminus gesprochene Sprache eine bestimmte Sprachverwendung. Dieser Terminus meint nicht, dass dem Gesprochenen und Geschriebenen verschiedene Sprachsysteme zugrunde liegen. Interviews folgen also primär den Regeln gesprochener Sprache, auch wenn sie nachträglich verschriftete Texte sind. Zweifellos geht im Prozess der Verschriftlichung und der Autorisierung der Interviews ein Teil der für die gesprochene Sprache typischen sprachlichen und nicht-sprachlichen Mittel verloren. Dennoch wäre es falsch anzunehmen, dass das nun schriftlich vorliegende Interview lediglich für geschriebene Sprache typische Formulierungen enthält, „da im Prinzip Merkmale sog. mündlicher Sprache auch in geschriebenen Texten bzw. umgekehrt Merkmale sog. geschriebener Sprache auch im mündlichen Sprachgebrauch gebildeter Kommunikationsteilnehmer vorkommen“ (Weigand 1993: 139).

Da Interviews mündlich zustande kommen, um danach abgedruckt zu erscheinen, ist die soziale Komponente kommunikativer Nähe und Distanz zu berücksichtigen. Die Bewertung von Textsorten kann im Sinne von Koch / Oesterreicher (1985) anhand einer Kreuzklassifikation nach dem Kriterium der (gesprochenen bzw. geschriebenen) Konzeption und dem des (graphischen bzw. phonischen) Mediums vorgenommen werden (vgl. Koch / Oesterreicher 1985: 18):

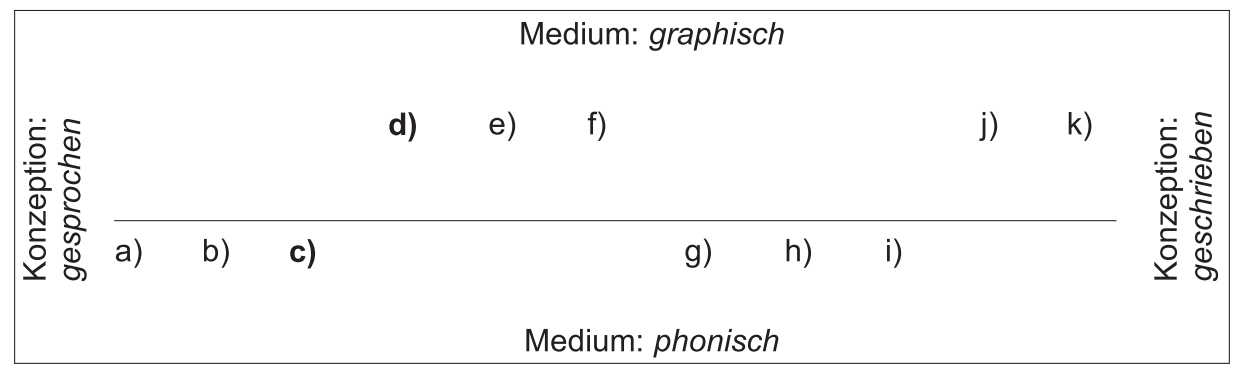

Abb. 1. Das Konzept von Koch / Oesterreicher (1985) 
Erläuterungen:

a) vertrautes Gespräch

b) Telefonat mit einem Freund

c) Interview

d) abgedrucktes Interview

e) Tagebucheintrag

f) Privatbrief

g) Vorstellungsgespräch

h) Predigt

i) Vortrag

j) FAZ-Artikel

k) Verwaltungsvorschrift

Diese Zusammenstellung macht den Unterschied zwischen einem Interview etwa im Rundfunk oder Fernsehen (c) und einem abgedruckten Interview (d) deutlich. Obwohl das Interview als solches konzeptionell gesprochen und medial phonisch realisiert wird, so erscheint das abgedruckte Interview konzeptionell zwar gesprochen, medial aber graphisch.

Im Zusammenhang mit dieser Klassifikation sprechen die Autoren dann auch von kommunikativer Nähe bzw. kommunikativer Distanz: „Es geht hier um das kommunikative Handeln der Gesprächspartner im Verhältnis zueinander und im Blick auf die sozialen, situativen und kontextuellen Gegebenheiten“ (vgl. Koch / Oesterreicher 2007: 350). Aus den Überlegungen beider Autoren ergeben sich folgende kommunikative Parameter für die Sprache der Nähe, die Eigenschaften der gesprochenen Sprache umfasst, und für die Sprache der Distanz, die für die geschriebene Sprache steht:

Tab. 1. Parameter der konzeptionellen Nähe und Distanz (vgl. Koch / Oesterreicher 2007: 351)

\begin{tabular}{|l|l|}
\hline \multicolumn{1}{|c|}{ Kommunikative Nähe } & \multicolumn{1}{c|}{\begin{tabular}{c}
\multicolumn{1}{c|}{ Extrem-Pol } \\
Kommunikative Distanz
\end{tabular}} \\
\hline - Privatheit & - Öffentlichkeit \\
- Vertrautheit der Kommunikationspartner & - Fremdheit der Kommunikationspartner \\
- starke emotionale Beteiligung & - geringe emotionale Beteiligung \\
- Situations- und Handlungseinbindung & - Situations- und Handlungsentbindung \\
- referenzielle Nähe & - referenzielle Distanz \\
- raum-zeitliche Nähe & - raum-zeitliche Distanz \\
- kommunikative Kooperation & - keine kommunikative Kooperation \\
- Dialogizität & - Monologizität \\
- Spontaneität & - Reflektiertheit \\
- freie Themenentwicklung & - Themenfixierung \\
\hline
\end{tabular}

Die von den Autoren vorgenommene Kreuzklassifikation sowie die angewandten Parameter machen weitere Unterschiede in der Einordnung der nicht ab- 
gedruckten und der im Druck veröffentlichten Interviews deutlich. Da der Gegenstand meiner Untersuchungen abgedruckte Interviews sind, charakterisiere ich sie in Anlehnung an das oben Skizzierte wie folgt:

- Abgedruckte Interviews sind konzeptionell mündliche und medial graphische Texte.

- Sie lassen sich nicht eindeutig einem Pol zuordnen und können daher als Mischtexte angesehen werden.

- Sie stehen zwar den Parametern kommunikativer Nähe näher, erfüllen aber einige Merkmale kommunikativer Distanz, wie etwa eine gewisse Themenfixierung und die Öffentlichkeit der Kommunikation.

\section{Textsorte Interview}

Allgemein kann als Ziel eines Interviews gelten, gewisse meist aktuelle Themen und Probleme unter besonderer Berücksichtigung der Meinung des Interviewten, seiner Argumente, Stellungnahmen und Begründungen darzustellen. Die zu untersuchenden Texte sind Medieninterviews, d.h. solche, die über Medien wie Presse, Rundfunk oder Fernsehen für ein breites Publikum veröffentlicht werden. Medieninterviews sensu largo lassen sich in Politiker-, Experten- und Starinterviews einteilen. Diesen drei Typen ist Mehrfachadressiertheit eigen. Ihr charakteristisches Merkmal ist, dass das im Interview Geäußerte durch den Leser bzw. Hörer bewertet wird. Dadurch haben sie ein besonderes Potential für die Selbstdarstellung des Interviewten. Als Möglichkeit zur Selbstdarstellung wird das Interview vor allem von Politikern und Prominenten genutzt, um sich recht ins Licht zu setzen (vgl. Schwitalla 1979: 182 f.). In Anlehnung an Schwitalla (1979: 164 ff.) lässt sich die uns hier interessierende Textsorte wie folgt charakterisieren:

- Presseinterviews sind eine dialogisierende Kommunikationsform.

- Presseinterviews mit Prominenten stellen eine Sprechsituation dar, in der eine Partei führend ist. In diesem Fall ist es der Interviewende, der dadurch privilegiert ist, dass er das Gespräch und dessen thematischen Verlauf steuert.

- Hinsichtlich der Mitteilungsperspektive gilt das Interview als vorzeitig bzw. nachzeitig darstellender Text, der durch Frage-Antwort-Sequenzen gekennzeichnet ist.

- In Interviews überwiegt die argumentative Themenentfaltung (mit deskriptiven Elementen). Dabei sollte m.E. keine Scheidung in allein argumentative und allein deskriptive Interviews vorgenommen werden, weil sich beide Eigenschaften in fast allen untersuchten Interviews überlappen.

- Medieninterviews mit Politikern oder Experten betreffen vorwiegend Bereiche öffentlichen Interesses, während sich Interviews mit Prominenten eher auf den nicht-öffentlichen, d.h. privaten Bereich und persönliche Angelegenheiten beziehen, für die ein gewisses Publikumsinteresse angenommen wird. 
Charakteristisch für das Interview ist der Umstand, dass es „eine einfache Redekonstellation [ist], meist zwei Gesprächspartner mit klaren Rollenzuweisungen und asymmetrischer Rangverteilung zur Steuerung des Dialogs" (Hess-Lüttich 1989: 184). In der Struktur des Interviews dominieren dementsprechend Fragen und Antworten als strukturprägende Handlungen. Die am zusammengestellten Material durchgeführten Untersuchungen zeigen deutlich, dass formelhafte Mittel besonders dem Vollzug von Antworthandlungen dienen. Der Grund dafür liegt darin, dass „Fragen kraftvolle Handlungen [sind], die erheblichen Druck ausüben. Zugleich sind Fragen das Paradebeispiel für initiative Sprechakte, die reaktive Akte („Antworten“ - darunter können u.a. fallen Begründen, Rechtfertigen, Behaupten, Erzählen, Bekennen) nahezu erzwingen“" (Sökeland 2003: 342).

\section{Analyse}

Einer pragmatischen Analyse wurden, wie oben vorausgeschickt, 25 Interviews mit Prominenten unterzogen. Ziel der Analyse war es, auf Formulierungsroutinen hinzuweisen, d.h. sprachliche Mittel, , die als mikrostrukturelle bzw. als makrostrukturelle Verfestigungen mental gespeichert sind und die als Formulierungsstereotype bzw. als Formulierungsmuster in Erscheinung treten" (Stein 1995: 301). In diesem Sinne verstehe ich nach Stein (1995) den Einsatz von Formeln als Formulierungsstrategie, um bestimmte pragmatische Aufgaben in der Kommunikation zu bewältigen. Ich gehe davon aus, dass Interviews musterhafte Texte sind, in denen sich zahlreiche Formeln und Routinemittel finden, die als typisch für diese Textsorte gelten.

Die durchzuführende Analyse berücksichtigt sowohl mehrgliedrige Formeln als auch einzelne Wörter, die für Interviews typisch sind, weil zweifelsohne auch Wörter wie ja, jaja oder Interjektionen $\ddot{a} h, h m$, die als Sprecher- und Hörersignale gelten, „formelhafte Züge tragen“ (Stein 1995: 81).

In allen journalistischen Interviews stehen das Fragen und Antworten im Vordergrund, weil ,das journalistische Interview klarer noch als andere Textsorten den Handlungscharakter der Sprache [belegt]: Seine geregelte Abfolge von Fragen und Antworten scheint wie geschaffen für eine sprechakttheoretische Analyse" (Sökeland 2003: 341). Dieses Rollenverhältnis bzw. der Rollenwechsel bedarf in einem Interview entsprechender Signalisierung. Sowohl das Fragen als auch das Antworten öffnen großen Spielraum für die Einführung von Formeln, die entweder den Text strukturieren oder die Einstellungen des Sprechers deutlich machen. Im Rahmen meiner Recherche konnte ich zwei Klassen formelhafter Ausdrücke aussondern:

- Dialogstrukturierende Formeln: Sie sind an bestimmte Situationen im Verlauf des Gespräches gebunden und werden in standardisierten Situationen verwendet. Sie stützen die Organisation und die Gliederung des Gespräches und haben eine wichtige Funktion in der Rollenverteilung zwischen dem Interviewer und 
dem Interviewten. Diese Formeln signalisieren einerseits die Rollenübernahmen, andererseits aber auch Widerspruch, Dissens oder - umgekehrt - Bestätigung.

- Einstellungssignale: Sie sind Mittel, mit denen in erster Linie die Einstellung und Intention des Sprechers signalisiert bzw. hervorgehoben werden.

Um die Darstellung kompakt zu halten, präsentiere ich im Folgenden kurze Belege zur Veranschaulichung, die durch den Interviewer in seinen Fragen (Kennzeichnung mit "Spiegel“) oder durch den Interviewten in seinen Antworten eingesetzt werden (ohne Kennzeichnung).

\subsection{Dialogstrukturierende Formeln}

\subsubsection{Formeln mit nein, nicht als Signale des Dissenses und Negierens}

Unter dem Begriff des Negierens verstehe ich nach Schatte / Schatte (2013: 100) „,[alle] Verfahren, etwas in Abrede zustellen“. Das Negieren dient dem Ausdruck eines Dissenses im Verlauf des Interviews, wobei dieser auf verschiedenen Ebenen erfolgen kann. „Will man dem Inhalt [Hervorhebung im Original - J.D.A.] einer vorausgehenden Partneräußerung widersprechen, daß heißt: will man behaupten, dass der von diesem wiedergegebene Sachverhalt nicht zutrifft" (Schatte / Schatte 2013: 112), so vollzieht man die Sprechhandlung Widersprechen. Dieses ist ein klares Signal für den Rollenwechsel und erfolgt mit der Antwortpartikel nein sowie ihren Kombinationen. Interessant ist, dass in unserem Material nur einmal allein die Partikel nein als selbständige Antwort auf die vorausgehende Frage des Interviewers verwendet wird. Vorwiegend wird sie durch ihr folgende Elemente oder eine ganze Äußerung ergänzt. Der Grund dafür scheint mir darin zu liegen, dass der Interviewte den Grund seines Widerspruchs ausführen möchte, um den Interviewer und Leser nicht mit einer schroffen Antwort zu konfrontieren und so für eine angemessene Partnerbeziehung zu sorgen. In dem von mir untersuchten Material sind folgende formelhafte Ausdrücke mit der Antwortpartikel nein enthalten:

\footnotetext{
Nein.

Nein, nein.

Nein, absolut nicht.

Nein, eigentlich nicht.

Nein! Noch schlimmer.

Nein, das ist faktisch falsch.

Nein, im Gegenteil [...].

Nein, ich will nur sagen [...].
}

Eine einfache Form, Widerspruch zu signalisieren, ist also der Einsatz der Partikel nein oder eventuell der Partikel doch, wenn die vorausgehende Frage des Interviewers negiert ist:

Doch, aber ungern. 
Eine andere Form des Negierens ist das Verneinen. Dieser Akt signalisiert keinen direkten Widerspruch dem Kommunikationspartner gegenüber. Er wird immer dann vollzogen, wenn der Sprecher ,in einer eigenen Äußerung einen Sachverhalt als nicht zutreffend bezeichnet" (Schatte / Schatte 2013: 117). Dieser Akt wird mit der Negationspartikel nicht vollzogen, was folgende in Antworten einzusetzende Formeln veranschaulichen:

Ich glaube nicht.

Ich weiß es nicht!

Das glaube ich nicht, im Gegenteil [...].

In den oben angeführten Belegen sind ganze Sätze negiert. Möglich sind auch elliptische Äußerungen als Formeln, in denen die Partikel nicht attribuiert ist:

Ganz und gar nicht.

Überhaupt nicht.

Ganz bestimmt nicht.

\subsubsection{Die reaktive Partikel $j a$ als Signal der Bestätigung}

Die Partikel ja kann zum Vollzug verschiedener sprachlicher Handlungen verwendet werden. Ihre primäre Funktion ist die Antwortpartikel ja. Sie dient als Antwort auf die vorausgehende Frage bzw. als Bestätigung der vorausgehenden Äußerung. In diesem Kontext fungiert sie ,partner-responsiv als Sprecherrollen-, Redeübernahme- und Rückmeldungssignal“ (Willkop 1988: 87). Das Hörersignal ja gewährleistet „,rasche Kontaktbestätigung“(Stein 1995: 189) sowie ungestörten Gesprächsfluss, indem der Sprecher seine Zustimmung nicht generell mit satzförmigen Antworten signalisieren muss. Das responsive $j a$ wird vorwiegend initial geäußert und nur selten nachgestellt. In unserem Material ist kein Beleg enthalten, in dem lediglich die Partikel ja als selbständige Antwort fungiert. Sie erscheint immer mit einem ihr folgenden Adverb oder mit einer explizierenden Äußerung. Möglich sind folgende formelhafte Ausdrücke mit ja:

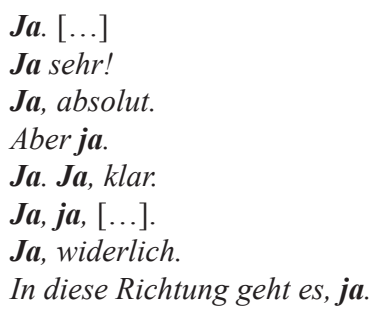

\subsubsection{Einschränkung der Zustimmung}

Im Folgenden möchte ich auf einige Formeln eingehen, die eingeschränkten Konsens signalisieren. Sie werden vom Sprecher verwendet, wenn er - evtl. in Rück- 
sicht auf den Partner - einen von diesem geäußerten Sachverhalt weder bestätigen noch ihm kategorisch widersprechen möchte:

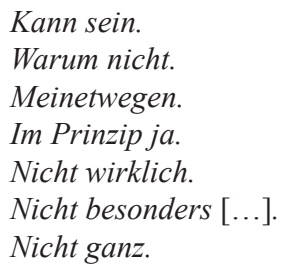

\subsubsection{Die Gliederungssignale also, na ja, tja}

Für Interviews ist die Partikel also als Quasi-Formel typisch. Sie signalisiert meist die Redeübernahme, kann aber auch als Indikator einer Paraphrase dienen (vgl. Willkop 1988: 136). Im Weiteren hilft sie, Formulierungsschwierigkeiten zu überwinden, indem sie dem Sprecher Zeitgewinn für die Versprachlichung seiner Erwiderung beschert:

Also, ich sehe das anders.

Also ja, überwiegend Männer.

Ausdrücke na ja, tja haben eine ähnliche Funktion wie also und werden von Weinrich (2007: 837) als Dialogpartikeln bezeichnet, die von der Zustimmung signalisierenden und bejahenden Partikel $j a$ weit entfernt sind. In den untersuchten Interviews mit Prominenten werden Interjektionen na ja (nun ja) und tja verwendet, um einerseits die Übernahme der Sprecherrolle, andererseits Nachdenklichkeit bzw. Absicherung des Sprechers zu signalisieren:

Na ja.

Tja.

Na ja, so Drogenrede.

Na ja. Es ist immer noch eine Schauspielerei.

Nun ja, ich mache eine Jubiläumssendung, weil die erste Folge jetzt 25 Jahre her ist.

\subsubsection{Unterbrechungssignal Entschuldigung}

Die Formel Entschuldigung betrachte ich anhand der Analyse der gesammelten Interviews nicht als echtes Höflichkeitssignal. In Interviews fungiert sie eher als Gliederungs- und als Strukturierungssignal, das dem Partner die Unterbrechung seines Redeflusses signalisiert. Durch den Einsatz dieser Formel kommt zusätzlich ein bestimmter Widerspruch zur Geltung:

Spiegel: Entschuldigung, aber Ihr Wortlaut war damals anders.

Entschuldigung, es ist gar nicht meine Absicht, die menschliche Existenz in allen ihren Dimensionen zu analysieren.

Entschuldigung, aber darauf wollten wir hinaus. 


\subsection{Einstellungssignale}

\subsubsection{Subjektive Bestätigungssignale seitens des Hörers}

Folgende formelhafte Ausdrücke dienen als Antworten und als Reaktion auf eine vorausgehende Entscheidungsfrage. In Dialogen werden Hörerbeiträge oft auf Ein-Wort-Äußerungen reduziert. Längere Formeln werden nur selten verwendet (vgl. Stein 1995: 190). Als subjektive Bestätigungssignale dienen vor allem Adverbien und Satzadverbien. Wie auch die reaktive Partikel $j a$ gelten sie als Bestätigungssignale. Dennoch ordne ich sie der zweiten Klasse zu, weil sie neben der bestätigenden Funktion die subjektive bzw. emotionale Einstellung des Sprechers zum Gesagten anzeigen:

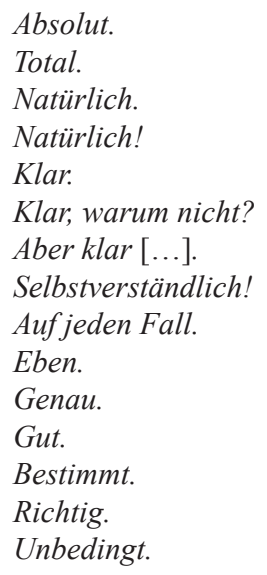

5.2.2. Die Abtönungspartikeln doch, ja, mal

Die vorgenommene Untersuchung von Presseinterviews zeigt, dass eine wichtige Funktion in ihnen den Abtönungspartikeln doch, ja und mal zukommt und sie als für Interviews formelhafte Mittel angesehen werden können. Die Partikeln ja und doch werden in Interviews am häufigsten verwendet. Sie spielen eine wichtige Rolle in der Argumentation, weil sie meist eine Begründung oder Erklärung für den in Rede stehenden Sachverhalt liefern. Sie referieren auf das den Partnern gemeinsame Wissen und verleihen den Argumenten des Interviewten bzw. des Interviewers einen bestimmten Nachdruck:

Das war immer gut, wir haben ja noch gemeinsam im Davis Cup gespielt.

Ich sage ja: Fußball wirkt Wunder.

Man kennt das ja aus der Jugend, wenn Eltern ihren Sohn unbedingt später mal im Nationaltrikot sehen wollen.

Ist es ja auch.

Spiegel: Zudem verdienen Sie ja auch noch die eine oder andere Million.

Ich lebe doch nicht auf den Cayman Islands. 
Seien Sie doch nicht immer so gehässig!

Spiegel: Als ältester Spieler unter den Top 100 kann man sich doch fragen: Wie lange tue ich mir das noch an?

Die Abtönungspartikel mal dagegen wird verwendet, um die Aufmerksamkeit des Hörers auf ein bestimmtes Anliegen zu richten. Sie ist von besonderer Bedeutung für die Herstellung des Partnerverhältnisses, indem sie Partnerzuwendung und Konsenssicherung signalisiert (vgl. Stein 1995: 206):

Nehmen Sie nur mal die Zahl der Ballwechsel.

Jetzt dürfen Sie mal raten!

\subsubsection{Das Überraschungssignal ach}

In gesprochener Sprache wie auch in verschrifteten Texten findet auch die Dialogpartikel ach Verwendung. Sie steht immer initial und signalisiert die Betroffenheit des Sprechers, die meist an einen gewissen Widerspruch, an Verwunderung oder Empörung über den gegebenen Sachverhalt oder die vorausgehende Äußerung gekoppelt ist. Mit diesem für Dialoge typischen Ausdruck „reagiert ein Dialogpartner auf einen für ihn unerwarteten Dialoginhalt" (Weinrich 2007: 837). Die Formel ach was indessen zeichnet aus, dass sich in ihr ,mit der Überraschung eine Nuance des Herunterspielens oder sogar der Geringschätzung [verbindet]“ (Weinrich 2007: 838):

Ach, kommen Sie.

Ach, nicht im Sinne, er war viel zu kurzsichtig.

Ach was!

\section{Zusammenfassung}

Die im vorausgehenden Abschnitt untersuchten Formeln sind in der folgenden tabellarischen Übersicht zusammengestellt:

Tab. 2. Dialogstrukturierende formelhafte Mittel

\begin{tabular}{|l|l|}
\hline \multicolumn{1}{|c|}{ Funktion } & \multicolumn{1}{|c|}{ ausgewählte Beispiele } \\
\hline Signal des Dissenses und Negierens & nein \\
& nein, nein \\
& nein, im Gegenteil \\
& Ich glaube nicht. \\
& ganz und gar nicht \\
& ganz bestimmt nicht \\
\hline
\end{tabular}




\begin{tabular}{|l|l|}
\hline Signal der Zustimmung & $\begin{array}{l}\text { ja } \\
\text { ja, ja } \\
\text { aber ja } \\
\text { ja sehr } \\
\text { ja absolut }\end{array}$ \\
\hline Einschränkung der Zustimmung & $\begin{array}{l}\text { kann sein } \\
\text { warum nicht } \\
\text { nicht wirklich } \\
\text { nicht ganz }\end{array}$ \\
\hline Gliederungssignale & $\begin{array}{l}\text { also } \\
\text { na ja } \\
\text { tja }\end{array}$ \\
\hline Unterbrechungssignal & Entschuldigung \\
\hline
\end{tabular}

Tab. 3. Einstellungssignalisierende formelhafte Mittel

\begin{tabular}{|l|l|}
\hline \multicolumn{1}{|c|}{ Funktion } & \multicolumn{1}{|c|}{ ausgewählte Beispiele } \\
\hline Subjektive Zustimmungssignale & $\begin{array}{l}\text { total } \\
\text { natürlich } \\
\text { selbstverständlich } \\
\text { genau } \\
\text { gut } \\
\text { richtig }\end{array}$ \\
\hline Abtönungspartikeln & $\begin{array}{l}\text { ja } \\
\text { doch } \\
\text { mal }\end{array}$ \\
\hline Überraschungs-/Empörungssignal & $\begin{array}{l}\text { ach } \\
\text { Ach, kommen Sie. } \\
\text { ach was! }\end{array}$ \\
\hline
\end{tabular}

Die oben dargestellten formelhaften Mittel betrachte ich als typisch für Presseinterviews. Sie geben den Kommunikationsteilnehmern die Möglichkeit, spontan und ohne größeren Aufwand Kommunikationsaufgaben wie Themawechsel, Textgliederung, Sprecher-Hörer-Wechsel oder Signalisierung der Sprecherintention zu bewältigen.

Die Grundstruktur des Interviews bilden Frage-Antwort-Sequenzen. Die meisten der untersuchten Mittel werden zum Vollzug der Sprechhandlung Antworten eingesetzt. So hat der Interviewte die Möglichkeit, seinen Dissens nicht allein mit der Partikel nein oder seine Zustimmung allein mit $j a$ einzubringen. Es wurde aufgezeigt, dass der Interviewte über ein Repertoire von Mitteln verfügt, um die Frage des Interviewers zu bejahen oder sie zu verneinen, und zwar in Abhängigkeit davon, wie er sich seinem Kommunikationspartner gegenüberstellen will oder 
wie weit er seine emotionale Anteilnahme preisgeben möchte. Die meisten Formeln haben primär textstrukturierende Funktion. Aus der Analyse folgt aber auch der Schluss, dass viele Formulierungen polyfunktional sind. So fungieren $a b s o l u t$, total, selbstverständlich als Einstellungssignale, zugleich aber auch als Zustimmungs- und Bestätigungssignale. Umgekehrt ist na ja ein Gliederungssignal, das zugleich Nachdenklichkeit oder Zweifel und damit eine bestimmte Einstellung anzeigt.

Die untersuchten Mittel können zweifellos in allen Arten von Interviews Verwendung finden. Der Vergleich von Interviews mit Experten und von solchen mit Politikern zeigt jedoch, dass Formeln wie ich weiß es nicht! / Ja, klar! / total, / ach was! / Ach, kommen Sie! oder Ausdrücke tja / absolut / total für Prominenten-Interviews charakteristisch sind. Das ist auf das m.E. wichtigste Merkmal von Interviews mit Prominenten zurückzuführen, das auf der hohen emotionalen Anteilnahme des Interviewten beruht, die in anderen Typen von Interviews im Hintergrund bleibt oder gehalten wird.

\section{Literatur}

Deppermann, Arnulf / Helmer, Henrike (2013): Standard des gesprochenen Deutsch: Begriff, methodische Zugänge und Phänomene aus interaktionslinguistischer Sicht. In: Hagemann, Jörg / Klein, Wolf Peter / Staffeldt, Sven (Hrsg.): Pragmatischer Standard. Tübingen. S. 111-141.

Feilke, Helmut (2012): Was sind Textroutinen? Zur Theorie und Methodik des Forschungsfeldes. In: Feilke, Helmut / Lehnen, Katrin (Hrsg.): Schreib- und Textroutinen. Frankfurt am Main. S. 1-31. Heinemann, Wolfgang / Vieweger, Dieter (1991): Textlinguistik. Eine Einführung. Tübingen.

Hess-Lüttich, Ernest W.B. (1989): Dialogsorten: Mediengespräche. In: Weigand, Edda / Hundsnurscher, Franz (Hrsg.): Dialoganalyse. Referate der 2. Arbeitstagung. Tübingen. S. 175-188.

Koch, Peter / Oesterreicher, Wulf (1985): Sprache der Nähe - Sprache der Distanz. Mündlichkeit und Schriftlichkeit im Spannungsfeld von Sprachtheorie und Sprachgeschichte. In: Romanistisches Jahrbuch XXXVI, S. 15-43.

Koch, Peter / Oesterreicher, Wulf (2007): Schriftlichkeit und kommunikative Distanz. In: Zeitschrift für germanistische Linguistik XXXV, S. 346-375.

Schatte, Christoph / Schatte, Czesława (2013): Negieren. In: Engel, Ulrich (Hrsg.): Argumentieren. Sprechen im deutsch-polnischen Kontrast. Dresden/Wrocław. S. 99-130.

Schwitalla, Johannes (1979): Dialogsteuerung in Interviews. Ansätze zu einer Theorie der Dialogsteuerung mit empirischen Untersuchungen von Politiker-, Experten- und Starinterviews in Rundfunk und Fernsehen. München.

Sökeland, Werner (2003): Das Interview - ein Frage-Antwort-Spiel? Zwölf Beobachtungen aus der journalistischen Praxis. In: Hagemann, Jörg / Sager, Sven F. (Hrsg.): Schriftliche und mündliche Kommunikation. Begriffe-Methoden - Analysen. Festschrift zum 65. Geburtstag von Klaus Brinker. Tübingen. S. 341-359.

Stein, Stephan (1995): Formelhafte Sprache. Untersuchungen zu ihren pragmatischen und kognitiven Funktionen im gegenwärtigen Deutsch. Frankfurt am Main.

Stein, Stephan (2004): Formelhaftigkeit und Routinen in mündlicher Kommunikation. In: Steyer, Katrin (Hrsg.): Wortverbindungen - mehr oder weniger fest. Berlin/New York. S. 262-288. 
Weinrich, Harald (2007): Textgrammatik der deutschen Sprache, Hildesheim/Zürich/New York.

Weigand, Edda (1993): Mündlich und schriftlich - ein Verwirrspiel. In: Weigand, Edda / Hundsnurscher, Franz: Dialoganalyse. Referate der 4. Arbeitstagung. Tübingen. S. 137-150.

Willkop, Eva-Marie (1988): Gliederungspartikeln im Dialog. München.

\section{Quellen}

Der Spiegel 3/2014: „Ich kann mich nicht quälen“ - Interview mit Tommy Haas

Der Spiegel 4/2014: „Kein Spaß, kein Gold“ - Interview mit Yao Ming

Der Spiegel 5/2014: „Lachen rettet uns“ - Interview mit Yasmina Reza

Der Spiegel 6/2014: „Das ist Bildungsfernsehen“ - Interview mit Frank Hoffmann

Der Spiegel 11/2014: „Es macht süchtig“ - Interview mit Nick Woodmann

Der Spiegel 15/2014: „So programmiert wie vor 20 Jahren“ - Interview mit Heinz Harald Frentzen

Der Spiegel 15/2014: „Klopp muss man verstehen“ - Interview mit Oliver Kahn

Der Spiegel 15/2014: „Künstliche Empörung“ - Interview mit Hans-Joachim Watzke

Der Spiegel 16/2014: „Linguistisch faszinierend “- Interview mit Jens Scholz

Der Spiegel 17/2014: „Verdrängen und abhacken“- Interview mit Gerhard Berger

Der Spiegel 22/2014: „Ich habe nie gefragt“ - Interview mit Adrew Ranicki

Der Spiegel 23/2014: „Wir dürfen nie nachlassen“ - Interview mit Cesare Prandelli

Der Spiegel 24/2014: „Der tiefe Punkt“ - Interview mit Joachim Löw

Der Spiegel 31/2014: „Ohrstöpsel sind unfair“ - Interview mit Lemmy Kilmister

Der Spiegel 34/2014: „Eine Art Lebenswerk“ - Interview mit Christoph Kramer

Der Spiegel 35/2014: „Ich bin der Drachentöter“ - Interview mit Boris Becker

Der Spiegel 37/2014: „Ich bin nun mal ungezogen“ - Interview mit Fatih Akin

Der Spiegel 38/2014: „Ist der Laden ausverkauft?“ - Interview mit Keith Richards

Der Spiegel 40/2014: „Das soll jetzt Sex sein?“ - Interview mit Lena Dunham

Der Spiegel 43/2014: „Ach, die Lippe. Funktioniert“ - Interview mit Billy Idol

Der Spiegel 44/2014: „Wir sind Junkies“ - Interview mit Jürgen von der Lippe

Der Spiegel 46/2014: „250 Essen pro Jahr“ - Interview mit Ralf Flinkenflügel

Der Spiegel 50/2014: „Es geht um nichts. Um Scheiß“ - Interview mit dem Rapper Haftbefehl

Der Spiegel 50/2014: „Für mich ist Journalismus eine Kunst“ - Interview mit Ulrich Matthes

Der Spiegel 50/2014: „Der rote Faden“ - Interview mit Joachim Löw

\section{Abstracts}

In dem vorliegenden Aufsatz wird versucht, das sprachliche Bild des »Der Spiegel«-Interviews mit Prominenten zu umreißen. Der Beitrag konzentriert sich dabei auf sprachliche und formelhafte Mittel, die für diesen Typ des Interviews prädestiniert sind. In dem Aufsatz wird auf das Modell der Sprache der Nähe und Distanz von Koch / Oesterreicher (1985) eingegangen. Des Weiteren werden die Eigenschaften der Textsorte Interview referiert. Anschließend wird eine Analyse der ausgewählten Formeln nach dem Kriterium ihrer pragmatischen Funktion vorgenommen. Der Aufsatz soll einen Beitrag dazu leisten, die sprachlichen Besonderheiten und Regularitäten des Textsortentyps Interview mit Prominenten zu erfassen.

Schlüsselwörter: Interviewsprache, formelhafte Sprache, Sprache der Nähe und Distanz, Formeln in Interviews 


\title{
Formulae in press interviews with celebrities illustrated by the magazine $»$ Der Spiegel $\ll$
}

The article is concerned with the linguistic picture of interviews with celebrities which appear in the German magazine »Der Spiegel«. The paper focuses on linguistic and formulaic lexical means predestined for this type of interview. The paper discusses the Koch's und Oesterreicher's model for language of immediacy and language of distance (1985). Secondly, the characteristics of the interview as a type of text are presented. In the next step the analysis of selected formulae is made according to their pragmatic function in the interview. The article shall contribute to illustrate the linguistic picture and certain language regularities in this type of text.

Keywords: interview language, formulaic language, language of immediacy and language of distance, formulae in interviews

\author{
Justyna Duch-Adamczyk \\ Uniwersytet im. Adama Mickiewicza w Poznaniu \\ Instytut Lingwistyki Stosowanej \\ Wydział Neofilologii \\ Al. Niepodległości 4 \\ 61-874 Poznań \\ Polen \\ E-Mail: jda@amu.edu.pl
}

